\title{
Meningococcal typing
}

The discovery in the last century that bacteria are important causes of human and animal disease and the realisation that bacteria with different properties cause different diseases was the key to the development of medical microbiology. Since then, much effort has been expended on the development and application of reliable methods for the identification and subdivision, or typing, of bacteria isolated from patients with disease. The ability to divide pathogenic species into distinct groups has permitted the development of epidemiology and enhanced the ability of medical science to recognise, treat and, in some cases, prevent bacterial diseases. Until recently the major tools used in bacterial typing were serological tests, relying on polyclonal sera raised in animals, and biochemical tests. However, over the last 10 years developments in molecular immunology and genetics have provided a wealth of new tools for bacterial typing. Perhaps more importantly, these technologies have provided detailed biochemical information that enables the development of new typing procedures.

Neisseria meningitidis, a major cause of bacterial meningitis and septicaemia, ${ }^{1,2}$ is an antigenically complex organism with a plethora of mechanisms that mediate changes in the antigens exposed on its surface. ${ }^{3}$ This presents several problems for typing. The ideal targets for bacterial typing reagents must vary sufficiently between isolates to provide epidemiologically useful discrimination, but must be sufficiently stable to be reliably distinctive. In practice, no single meningococcal antigen or gene provides all the information necessary for the reliable typing of isolates.

The earliest subdivision of meningococci was achieved with polyclonal antisera that divided the species into serogroups, which were designated by capital letters (A, B, C etc); it is now known that these serogroups are based on the serological reactivity of the capsular polysaccharide. ${ }^{4}$ Chemical analyses have determined the structure of these polysaccharide antigens and monoclonal antibody (MAb) reagents are now available for most of the serogroups. In many laboratories around the world, serogrouping remains the most detailed level of identification of meningococcal isolates available. There are nine recognised serogroups, but of these only five-A, B, C, and to a lesser extent $\mathrm{Y}$ and $\mathrm{W}-135$-commonly cause disease; however, there is much diversity among meningococci of the same serogroup and more detailed information is required to designate strains precisely. This is particularly important as there is a geographical bias in the occurrence of meningococcal serogroups. In the $\mathrm{UK}$, for example, most isolates are either serogroup B or $\mathrm{C}$, whereas in the meningitis belt of Africa disease is usually caused by serogroup A organisms.

The serogroups of $N$. meningitidis are subdivided, on the basis of the immune reactivities of their outer membranes, into serotypes, serosubtypes and immunotypes. The serotyping antigens are the mutually exclusive class 2 and 3 outer-membrane proteins (OMPs) and the serosubtyping antigen is the class 1 OMP. ${ }^{5}$ Immunotypes are defined by the lipooligosaccharide (LOS), which is the glycolipid component of the outer membrane. ${ }^{6}$ Other surface antigens such as pili and opacity proteins (Opas) often vary within one isolate during growth in vivo and in vitro, and consequently are not suitable as typing targets.? Meningococcal isolates are also distinguished phenotypically by multilocus enzyme electrophoresis

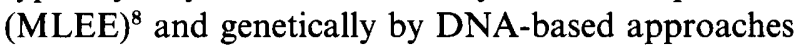
such as restriction fragment length polymorphism (RFLP) analyses, pulsed field gel electrophoresis (PFGE) fingerprinting, and ribotyping. ${ }^{9-11}$

During the 1980s, the application of MAb technology permitted the development of highly specific meningococcal typing reagents. ${ }^{12}$ Recent molecular analyses have revealed the structural features of the OMPs that define their interactions with the MAbs used for typing. The class 1, 2 and 3 OMPs are related porins, or pore-forming proteins. These proteins have loops, of variable length and amino-acid sequence, that extend from the cell surface. In the case of the serosubtypes, short continuous epitopes recognised by the MAbs are located in these loops. ${ }^{13-15}$ However, some of the serosubtype epitopes, and most of the serotype epitopes, are not easily defined in terms of a single, short peptide sequence, and these epitopes may be discontinuous, comprising amino acids from more than one surface loop.

Detailed genetic studies based on both MLEE and, particularly, on nucleotide sequence analyses, have exposed some weaknesses in this scheme for the typing of meningococci. These problems fall into two categories: (i) those associated with the population genetics of the organism, and (ii) those associated with the $\mathrm{MAb}$ reagents.

$N$. meningitidis is a transformable organism; this means that it is able (competent) to take up DNA from the environment and incorporate it into its own genome. Thus, a mechanism for the sexual transfer of genes encoding antigens is available to the meningococci and there is increasing evidence that this process 
occurs frequently, especially in the meningococci of serogroup B and $\mathrm{C} .{ }^{16}$ The genetic information encoding the target antigens (capsule, OMPs, LOS etc.) can be transferred easily between strains making typing based on a single antigen unreliable, particularly when that antigen is likely to be subject to selective pressure imposed by the host's immune system. Therefore, meningococci should be typed by an approach that involves the examination of combinations of antigens, preferably encoded by several unlinked genetic loci. Genotypic data, such as that obtained by PFGE or RFLP analyses, enhance the information obtained by analysis of single genes or gene products.

Although most national reference centres have reagents for serogrouping, no single standard set of reagents is available. The situation is better for serotype and serosubtype analysis, as a kit consisting of specific MAbs has been available for several years. However, most of the MAbs against the OMPs were generated before the elucidation of the primary structures of proteins. Molecular analyses have demonstrated that the reagents are not comprehensive for two reasons. First, there are many variants of the proteins that are not recognised by the MAbs, and second, when there is an antibody that reacts with a particular variant, sequence analyses have revealed sub-variants that react unpredictably with the MAb. In the worst case, it is possible for two strains to have identical genomes except for a single base change that alters the amino-acid sequence recognised by the $\mathrm{MAb}$. Thus two serologically distinct strains will be almost identical genetically.

There are two ways of improving the current techniques by the use of DNA technology--(i) developing new systems for studying current targets and (ii) using DNA approaches to identify new targets. For

\section{References}

1. Schwartz B, Moore PS, Broome CV. Global epidemiology of meningococcal disease. Clin Microbiol Rev 1989; 2 Suppl: s118-s124.

2. Peltola H. Meningococcal disease: still with us. Rev Infect Dis $1983 ; 5: 71-91$.

3. Poolman JT, Hopman CTP, Zanen HC. Problems in the definition of meningococcal serotypes. FEMS Microbiol Lett 1982; 13: 339-348

4. Vedros NA. Development of meningococcal serogroups. In: Evolution of meningococcal disease, vol 2. Boca Raton, Florida, CRC Press Inc. 1987: 33-37.

5. Tsai C-M, Frasch CE, Mocca LF. Five structural classes of major outer membrane proteins in Neisseria meningitidis. $J$ Bacteriol 1981; 146: 69-78

6. Tsai C-M, Mocca LF, Frasch CE. Immunotype epitopes of Neisseria meningitidis lipooligosaccharide types 1 through 8. Infect Immun 1987; 55: 1652-1656.

7. Frasch CE, Zollinger WD, Poolman JT. Serotype antigens of Neisseria meningitidis and a proposed scheme for designation of serotypes. Rev Infect Dis 1985; 7: 504-510.

8. Caugant DA, Frøholm LO, Bøvre $\mathbf{K}$ et al. Intercontinental spread of a genetically distinctive complex of clones of Neisseria meningitidis causing epidemic disease. Proc Natl Acad Sci USA 1986; 83: 4927-4931.

9. Bygraves JA, Maiden MCJ. Analysis of the clonal relationships between strains of Neisseria meningitidis by pulsed field gel electrophoresis. J Gen Microbiol 1992; 138: 523-531. example, a scheme for subtyping meningococci, based upon the use of the polymerase chain reaction (PCR), has been developed. ${ }^{17}$ To determine the subtype of a meningococcal isolate the gene (por $A$ ) encoding the class 1 OMP, amplified by the PCR, is labelled and used to probe reference DNA samples immobilised in an ordered array on a membrane filter. The reference samples consist of bacteriophage M13 DNA preparations, each containing a cloned fragment of porA encoding a particular variable loop of the class 1 OMP. This scheme is completely analogous to subtyping with MAbs, for it is similarly directed against the variability of the class 1 OMP; however, it detects the variation in the DNA encoding the class 1 OMP rather than the variation in the antigen itself. Consequently, this approach has several advantages: more subtypes are detected than by the current range of MAbs; new subtypes are easily added to the system; and the standard DNA samples can be produced and distributed quickly and cheaply to reference laboratories.

Meningococcal meningitis remains an important health problem throughout the world against which no comprehensive vaccine is available. The design and evaluation of improved vaccines is crucially dependent on the availability and quality of epidemiological data which, in the case of the meningococci, has been based largely upon serological analyses. These analyses, in spite of their shortcomings, have provided a sound basis for studying the epidemiology of meningococci, but modern molecular genetic techniques now afford rapid, reliable and robust alternatives to serological approaches.

M. C. J. Maiden and I. M. Feavers

Division of Bacteriology, National Institute for Biological Standards and Control, Blanche Lane, South Mimms, Potters Bar, EN6 3QG.

Correspondence should be sent to Dr I. M. Feavers.

10. Fox AJ, Jones DM, Gray SJ, Caugant DA, Saunders NA. An epidemiologically valuable typing method for Neisseria meningitidis by analysis of restriction fragment length polymorphisms. J Med Microbiol 1991; 34: 265-270.

11. Jordens JZ, Pennington TH. Characterization of Neisseria meningitidis isolated by ribosomal RNA gene restriction patterns and restriction endonuclease digestion of chromosomal DNA. Epidemiol Infect 1991; 107: 253-262.

12. Abdillahi H, Poolman JT. Whole-cell ELISA for typing Neisseria meningitidis with monoclonal antibodies. FEMS Microbiol Lett 1987; 48: 367-371.

13. McGuinness B, Barlow AK, Clarke IN et al. Deduced amino acid sequence of class 1 protein (Por $A$ ) from three strains of Neisseria meningitidis. J Exp Med 1990; 171 : 1871-1882.

14. Maiden MCJ, Suker J, McKenna AJ, Bygraves JA, Feavers IM. Comparison of the class 1 outer membrane proteins of eight serological reference strains of Neisseria meningitidis. Mol Microbiol 1991; 5: 727-736.

15. van der Ley $\mathrm{P}$, Heckels JE, Virji M, Hoogerhout P, Poolman JT. Topology of outer membrane porins in pathogenic Neisseria spp. Infect Immun 1991; 59: 2963-2971.

16. Maiden MCJ. Population genetics of a transformable bacterium: the influence of horizontal genetical exchange on the biology of Neisseria meningitidis. FEMS Microbiol Lett 1993; 112: 243-250.

17. Maiden MCJ, Bygraves JA, McCarvil J, Feavers IM. Identification of meningococcal serosubtypes by polymerase chain reaction. J Clin Microbiol 1992; 30: 2835-2841. 